\title{
Haemolytic anaemia as an initial presentation of Wilson's disease in paediatric patients
}

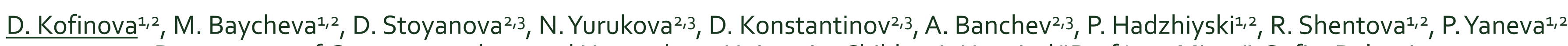
'Department of Gastroenterology and Hepatology, University Children's Hospital "Prof Ivan Mitev", Sofia, Bulgaria ${ }^{2}$ Medical University of Sofia, Bulgaria

3Department of Paediatric Haematology and Oncology, University Hospital "Tsaritsa Yoanna", Sofia, Bulgaria

- Objectives and study: Wilson's disease is a rare autosomal recessive genetic disorder of copper metabolism, causing copper induced damage to various organs. The clinical presentation is highly diverse. Although hepatic disease is the common presentation in children and adolescents, neurologic, psychiatric, and haematological symptoms do occur. Haemolytic anaemia is a rare clinical manifestation of Wilson's disease in up to $20 \%$ (1).

Methods: We report three cases of Wilson's disease presented with haemolytic anaemia and liver involvement.

* Patient 1, 8-year-old boy presented with nausea, vomiting, abdominal pain, jaundice, dark urine and pale stools. Physical examination revealed hepatoslenomegaly, normal neurological status and Kayser-Fleischer rings in both eyes. The laboratory tests showed Coomb's negative haemolytic anaemia, mildly elevated liver enzymes, both conjugated and unconjugated hyperbilirubinemia, hypoalbuminemia with coagulation failure. The diagnosis was genetically confirmed after registering low ceruloplasmin and high urinary copper excretion after penicillamine challenge.

* Patient 2, 7-year-old girl complained of fatigue, weight loss, abdominal pain, jaundice and dark urine. She had hepatomegaly and Kayser-Fleisher ring in the eyes with no neurological symptoms. Jaundice has reached extreme values of bilirubin up to $1892 \mu \mathrm{mol} / \mathrm{l}$. Ceruloplasmin was normal, but the penicillamine challenge showed elevated copper in the urine. Genetic analysis confirmed Wilson's disease.

* Patient 3, 16-year-old girl presented with jaundice and dark urine. She had hepatoslenomegaly, mild tremor and Kayser-Fleisher ring in the eyes. Ceruloplasmin was low and urinary copper excretion after penicillamine challenge was high. The genetic test showed she has compound heterozygous mutations.

$>$ All patients showed Coombs-negative haemolytic anaemia with high reticulocytes rate and liver failure. All of them were tested for viral hepatitis and were negative. They are treated with penicillamine $20 \mathrm{mg} / \mathrm{kg}$ and have normal liver function one year later. Patient 3 is being transitioned and followed up by an adult hepatologist.

\begin{tabular}{|c|c|c|c|c|c|}
\hline & $\begin{array}{l}\mathrm{Hgb} \\
\mathrm{g} / \mathrm{l}\end{array}$ & $\begin{array}{l}\mathrm{RBC} \\
\times 10^{12}\end{array}$ & $\begin{array}{l}\text { MCV } \\
\mathrm{fl}\end{array}$ & $\begin{array}{l}\text { Retic } \\
\% 0\end{array}$ & Coomb's test \\
\hline Patient 1 & 38 & 1.07 & 107 & 93 & Negative (-) \\
\hline Patient 2 & 51 & 1.5 & 113 & 65 & Negative (-) \\
\hline Patient 3 & 68 & 1.9 & 109 & 70 & Negative (-) \\
\hline & & \multicolumn{2}{|c|}{$\begin{array}{l}\text { Hepatomegaly } \\
\text { (under the rib) }\end{array}$} & \multicolumn{2}{|c|}{$\begin{array}{l}\text { Splenomegaly } \\
\text { (under the rib) }\end{array}$} \\
\hline Patient 1 & & \multicolumn{2}{|l|}{$2 \mathrm{~cm}$} & \multicolumn{2}{|c|}{$3,5 \mathrm{~cm}$} \\
\hline Patient 2 & & \multicolumn{2}{|l|}{$5 \mathrm{~cm}$} & \multicolumn{2}{|c|}{ Non palpable } \\
\hline Patient 3 & & \multicolumn{2}{|l|}{$5 \mathrm{~cm}$} & \multicolumn{2}{|c|}{$3 \mathrm{~cm}$} \\
\hline
\end{tabular}

\begin{tabular}{l|l|l|} 
& $\begin{array}{l}\text { Neurological } \\
\text { symptoms }\end{array}$ & Kayser-Fleischer ring \\
\hline Patient 1 & Negative (-) & Positive (+) in both eyes \\
\hline Patient 2 & Negative (-) & Positive (+) in both eyes \\
\hline Patient 3 & Mild tremor & Positive (+) in both eyes
\end{tabular}

\begin{tabular}{l|l} 
& Mutation \\
\hline Patient 1 & $\mathrm{H} 1069 \mathrm{O} / \mathrm{H}_{1069 O}$ \\
\hline Patient 2 & $\mathrm{C} .1708-1 \mathrm{G}>\mathrm{A} / \mathrm{C} .1708-1 \mathrm{G}>\mathrm{A}$ \\
Patient 3 & $\mathrm{H} 1069 \mathrm{O} / \mathrm{P} 1134 \mathrm{Pfs}$
\end{tabular}

\begin{tabular}{|c|c|c|c|c|c|c|c|c|c|c|c|}
\hline & $\begin{array}{l}\text { ASAT } \\
\text { IU/I }\end{array}$ & $\begin{array}{l}\text { ALAT } \\
\text { IU/I }\end{array}$ & $\begin{array}{l}\text { ALP } \\
\mid \mathrm{IU} / \mathrm{I}\end{array}$ & $\begin{array}{l}\text { GGT } \\
\text { IU/I }\end{array}$ & $\begin{array}{l}\text { Bilirubin } \\
\text { umol/l }\end{array}$ & $\begin{array}{l}\text { Direct } \\
\text { bilirubin } \\
\text { umol/l }\end{array}$ & $\begin{array}{l}\text { Protein } \\
\text { g/l }\end{array}$ & $\begin{array}{l}\text { Albumin } \\
\text { g/l }\end{array}$ & INR & $\begin{array}{l}\text { Ceruloplasmin } \\
\text { (N 0.2-0.6) } \\
\text { g/l }\end{array}$ & $\begin{array}{l}\text { Penicillamine } \\
\text { challenge } \\
\text { umol/l }\end{array}$ \\
\hline Patient 1 & 125 & 16.7 & 18.6 & 72.7 & 213.7 & 132.7 & 53 & 29 & 3.0 & 0.18 & 43.1 \\
\hline Patient 3 & 79.9 & 32.8 & 31 & 206 & 778 & 670.4 & 60 & 29 & 2.43 & 0.14 & 45 \\
\hline
\end{tabular}

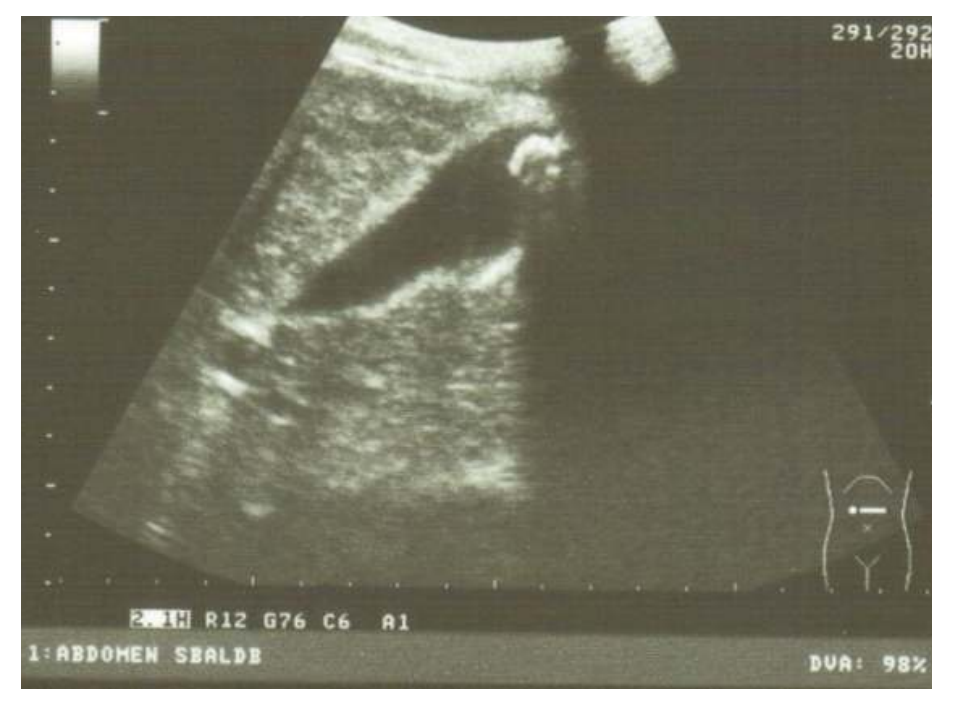

Patient 1 - Enlarged liver heterogeneous with gallstones

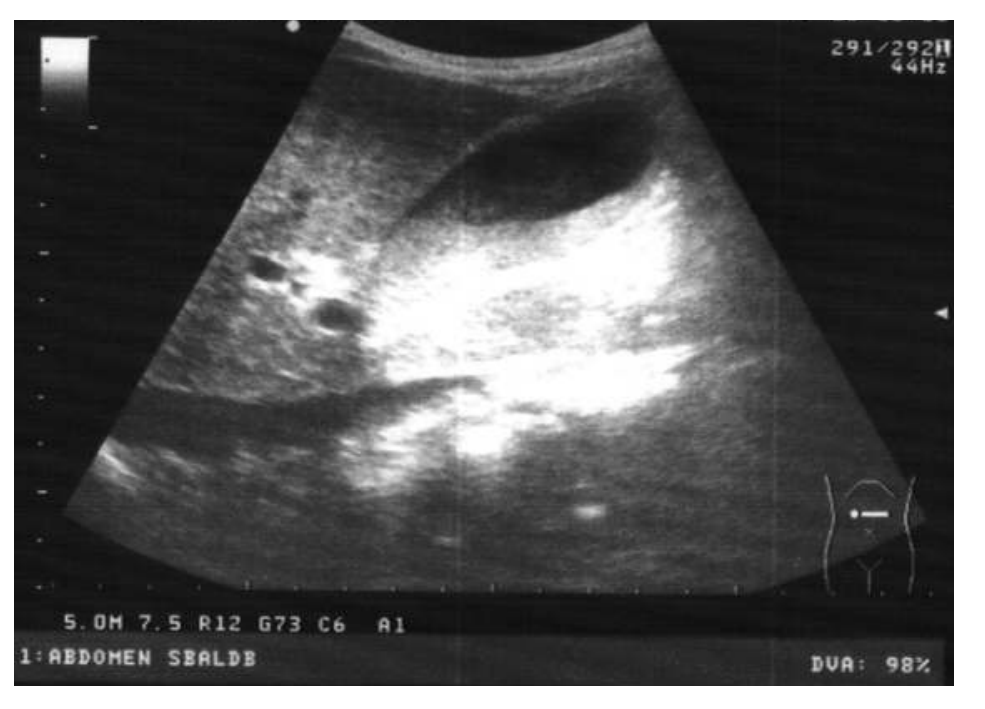

Patient 2: Enlarged hyperechoic liver with gallbladder with sludge

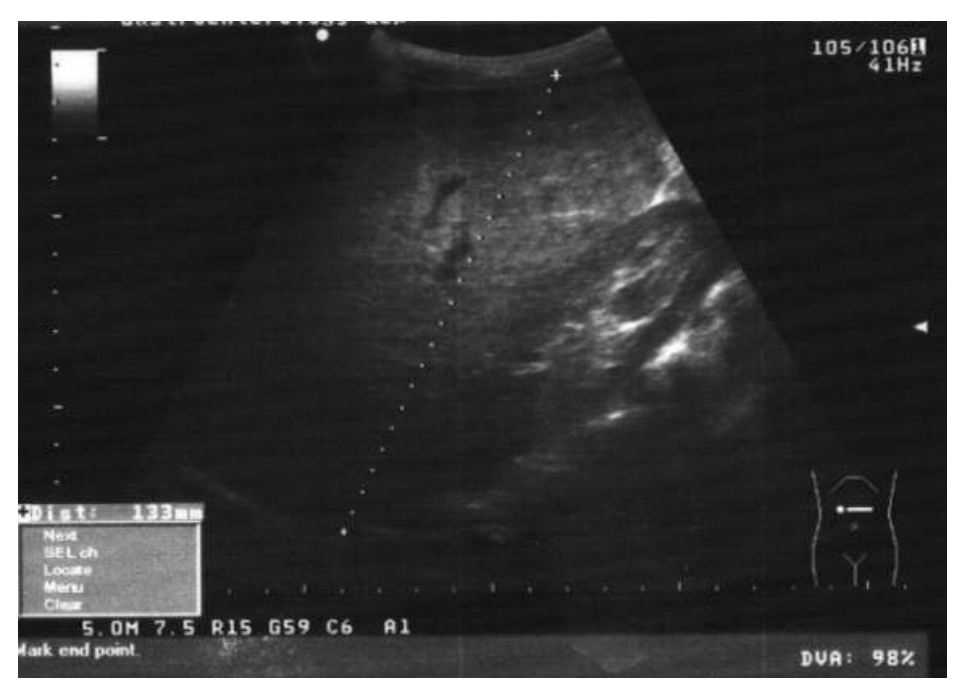

Patient 3 - Enlarged hyperechoic liver, cirrhosis

Conclusion: Wilson's disease should be considered in paediatric patients presenting with haemolytic anaemia, liver dysfunction or neurological symptoms of unknown cause. Establishing diagnosis and starting an appropriate treatment can be life-saving. 